\title{
Design of Marine Geological Survey Monitoring Model and Its Realization
}

\author{
CHEN Hong-Wen ${ }^{1,}$ a , SHAO Chang-gao ${ }^{2, b}$, \\ MLR Key Laboratory of Marine Mineral Resources, Guangzhou Marine Geological \\ Survey, Guangzhou 510075,China \\ achw@hydz.cn, bscg@hydz.cn
}

Keywords: Geological Survey project, Monitoring Model, Visualization.

Abstract:The marine geological survey project is an important method of marine data acquisition, which relates to the national marine rights and interests and security. To strengthen monitoring and dispatching of survey ships and supervision on offshore projects, a marine geological survey monitoring model, based on satellite communication system and WEBGIS, is developed, which Contrast the ship's real-time position, the survey line and station information about the plan. Practical application shows that the model improves the supervision level of marine geological survey project and reduces project risks.

\section{Introduction}

Marine Geological Survey is significant, which is the basis of the construction of the harbor and exploitation of undersea resources. In order to effectively to protect national maritime rights and interests, rationally exploit of marine resources and protect the marine environment, marine geological survey project management is now imminent.

Over the years, the supervision of our marine geological survey project always stays in the primitive stage, Only a few words are sent to describe the progress of project by email or fax. In this way the ship's position and the progress of the project is hard to be described clearly, which leads to be impossible to accurately grasp the project. Deeply studied the static structure model and dynamic behavior model of marine geological survey project,the monitoring model of the project was designed to evaluate and supervise project, which Contrasts the ship's real-time position, the survey line and station information to the plan. Practical application shows that the model improves the supervision level of marine geological survey project and reduces project risks.

\section{The static structure model of geological survey project}

The static structure model was expressed by Unified Modeling Language (UML), which is a general-purpose modeling language in the field of software engineering, that is designed to provide a standard way to visualize the design of a system. ${ }^{[1]}$ Structure diagrams emphasize the things that must be present in the system being modeled. Since structure diagrams represent the structure, they are used extensively in documenting the software architecture of software systems. For example,

the component diagram which describes how a software system is split up into components and shows the dependencies on these components.

The Project static structure model is mainly composed of the work area classes of investigation, the survey line classes and the geological sampling station classes. All involved core elements and their relationship is shown in Fig. 1. The investigation project classes is core category of the static structure, which can correspond to one or more working area. A working area can also correspond to a plurality of survey lines classes and geological sampling station classes. 


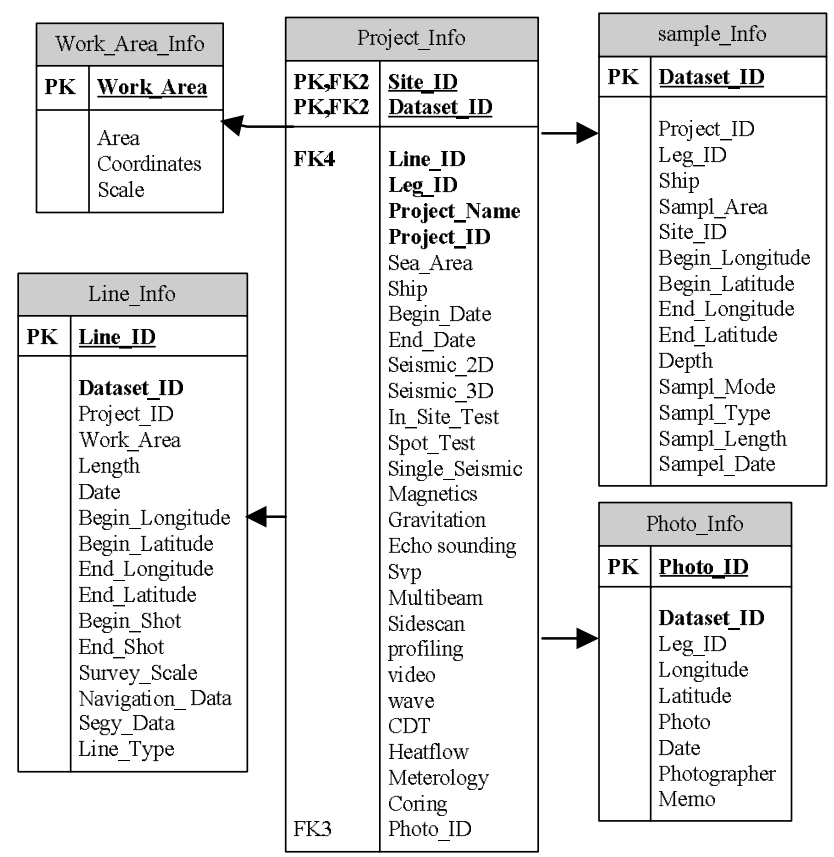

Fig.1 The static structure model of geological survey project

\section{Interaction diagrams model of geological survey project}

The static structure model of geological survey project reveals attributes and interface of the four core classes. The interaction diagrams of Geological Survey project, a subset of behavior diagrams, emphasize the flow of control and data among the things in the system being modeled.

The interactive operation sequence relationships among the project, work area classes of investigation, the survey line classes and geological sampling station classes is shown in Fig.2.

First of all, a new survey project needs to be created and then edited the properties of the project. Secondly, the working area of the project needs to be created, and imported range of working area on map and edited the properties of the area. Thirdly, the survey line and geological sampling station needs to be created just as working area. From now on, Construction plan of the project was complete imported into database. Along with the progress of the project, the progress report, which should contain survey line information and geological sampling station information, was imported into the database. By matching the line number and the station number, project progress was calculated and the map was updated.

As a management way of marine geological survey project management, the earned value analysis is the main measurement index in the project schedule and cost control, and it plays an important role in schedule management and cost management. The analysis can forecast the likely cost to complete the project and adjusting to the project plan. 


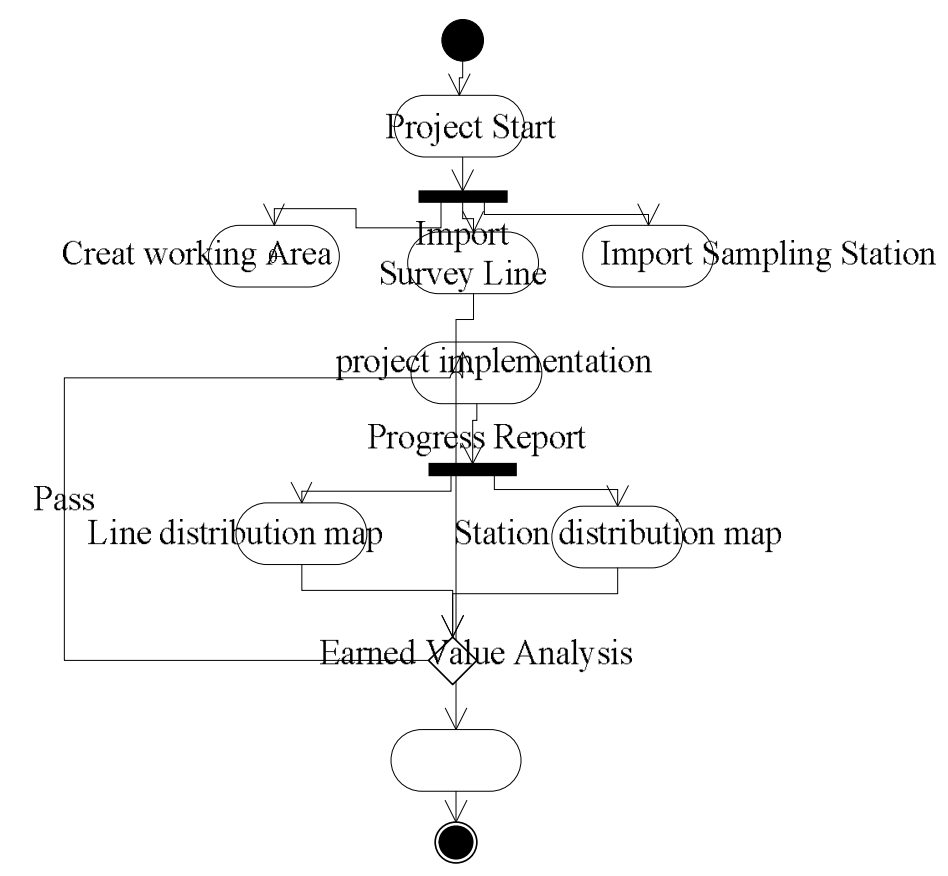

Fig. 2 Activity diagrams model of geological survey project

\section{Automatic graphic and comparative analyses}

This model, on the basis of analyzing project progress report, can get access to the position of ship and record its path by mapping in the chart. As shown in Fig3, the red line indicates that the line has been completed, and the green line is not complete.

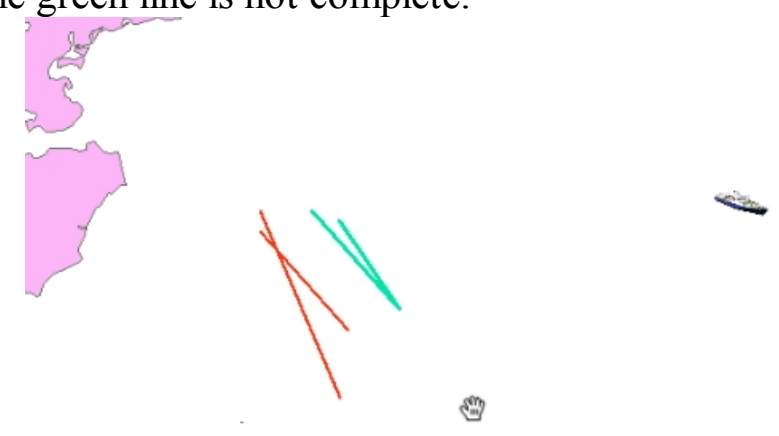

Fig.3 Distribution map of the Survey Line

\section{Model realization}

Introduce a research of marine geological survey monitoring system based on WebGIS model, which used ArcGIS Server as its Geographic Information System server. It has the advantages of managing the geographical figure and spatial data.

Analyzed evaluation model, the marine geological survey project is according to certain rules, which are made up of a series of activities. The evaluation of survey line and geological sampling station is the key to the evaluation of the project. The algorithm of survey line earned value analysis is as follow: $\mathrm{CEV}=(c a c / c p v) /(p t-c t)$

Where the cac is the kilometers of survey line that has been completed; the cpv is the kilometers of survey line that is unfinished; the pt is planned duration of the project; the ct is Operation duration.

The algorithm of geological sampling station earned value analysis is as follow:

$\mathrm{PEV}=(p a c / p p v) /(p t-c t)$ 
Where the pac is the count of station that has been completed; the cpv is the count of station that is unfinished. Core code is as follows:

public String voyage Statistics() \{

StringBuffer str $=$ new StringBuffer();

str $=$ str.append $("<\operatorname{root}>")$;

List $<$ VoyagePlan $>$ list $=$ getAllVoyagePlan();

for (int $\mathrm{i}=0 ; \mathrm{i}<$ list.size ()$; \mathrm{i}++)\{$

VoyagePlan $v p=$ list.get(i);

str = str.append("<voyage vNO=" + "'" + vp.getVoNo() + "'" >");

List $<$ StationInfo $>$ SIlist $=$ this.findStationByVoNo(vp.getVoNo());

List $<$ StationPlan $>$ SPlist $=$ this.findStationPlanByVoNo(vp.getVoNo());

List $<$ SurveyPlan $>$ SPLlist $=$ this. findSurveyPlanByVoNo(vp.getVoNo());

List $<$ SurveyLine $>$ SLlist $=$ this.findSurveyLineByVoNo(vp.getVoNo());

SurveyPlan sPlan = null;

double spCount $=0.0$;

for (int $\mathrm{j}=0 ; \mathrm{j}<\mathrm{SPlst}$.size ()$; \mathrm{j}++)\{$

sPlan $=$ SPlst.get $(\mathrm{j})$;

spCount $+=$ Double.parseDouble(sPlan.getPlanLength ()$) ; \quad$ \}

SurveyLine sLine $=$ null;

double slCount $=0.0$;

for (int $\mathrm{j}=0 ; \mathrm{j}<$ SLlist.size ()$; \mathrm{j}++)\{\quad$ sLine $=$ SLlist.get $(\mathrm{j})$;

slCount $+=$ Double.parseDouble(sLine.getActualLength()); $\}$

str = str.append $("<$ projectNo vNO=" + "'" + SPlist.size ()$+$ +"' + "+ " / $>$ ");

str $=$ str.append $("<$ stationPlan $\mathrm{vNO}="+$ +"' + SIlist.size ()$+$ "'" + "'"+ " />");

str $=$ str.append $("<$ stationInfo vNO=" + "'" + spCount $+" '+$ + " />");

str = str.append $("<$ surveyPlan vNO=" + "'" + slCount + "'" " />");

str $=$ str.append $("</$ voyage $>") ;\}$

str $=$ str.append $("</$ root $>")$;

return str.toString ()$; \quad\}$

\section{Conclusions}

Studied the static and dynamic behavior of marine geological survey project, a monitoring model of the project is accomplished in this paper, which has been realized in Guangzhou Marine Geological Survey (GMGS). This model shows us directly the progress of the project and the position of the ship. All these ensure the safety of offshore production operation and shipping facilities.

\section{Acknowledgements}

This work is supported by the national special funds (GZH201100312-02).

\section{References}

[1] [1]Unified Modeling Language User Guide, The (2 ed.). Addison-Wesley. 2005. p. 496. ISBN 0321267974.

[2] FENG Bin, TAN Jian-jun, LI Shao-rong:Design and Implementation of DBMS for Marine Geological Survey. Computer Engineering. Vol.35, No. 3 (2009),p.29 ( in Chinese ) . 